\title{
The role of some spatial parameters of gratings on the McCollough effect*
}

\author{
JOHN J. UHLARIK $\uparrow$ and ALAN G. OSGOOD \\ Kansas State University, Manhattan, Kansas 66506
}

\begin{abstract}
Ss were alternately adapted to vertical and horizontal gratings that consisted of black bars and colored slits. The slits of one grating were green and of the other, magenta. The widths of the black bars and the colored slits were varied independently during adaptation and testing. This design separates the relative influence of bar width, slit width, and spatial frequency on an orientation specific color aftereffect known as the McCollough effect. Black bar width had the major influence on the strength of the aftereffect, suggesting that the neurophysiological mechanism underlying the McCullough effect might consist of orientation specific units that are sensitive to both the widths of black bars and the chromatic characteristics of their surrounds.
\end{abstract}

McCollough (1965) demonstrated a pattern (orientation) contingent color aftereffect. The aftereffect was produced by alternate exposure to horizontal and vertical striped gratings. One grating consisted of black bars and blue slits and the other, black bars and orange slits. After exposure to these adapting gratings, observers viewed black and white striped test gratings and reported complementary color aftereffects that were specific to the orientation of the test gratings. Other investigators have reported similar findings for other combinations of colored and black gratings (e.g., Stromeyer, 1969).

The number of repetitions of black and colored stripes per unit visual angle, hereafter referred to as spatial frequency, affects the strength of the McCollough effect (Teft \& Clark, 1968; Stromeyer, 1972). In addition, Harris (1970) demonstrated that a pattern (frequency) contingent color aftereffect, similar to the orientation specific McCullough effect, was strongest when the spatial frequency of the test grating matched that of the adapting gratings. Furthermore, Harris's study showed that the strength of the aftereffect was dependent upon the retinal width subtended by a given spatial frequency rather than upon perceived width. Three spatial parameters of the gratings were confounded both during adaptation and testing for the aftereffect in the studies of spatial frequency referred to above. These parameters were (a) width of the black bars, (b) width of the colored (or white) slits, and (c) spatial frequency. Thus, it has not been possible to separate the relative contributions of these parameters to the McCollough effect.

Harris (1971) pointed out the importance of

* This research was supported by funds from Grant MH 20862-01 A1 from the National Institute of Mental Health and Grant F-40 from the Bureau of General Research, Kansas State University. The authors would like to thank Gary Wells for his assistance in conducting the experiment.

+Requests for reprints should be sent to John J. Uhlarik, Department of Psychology, Kansas State University, Manhattan, Kansas 66506 . separating these three spatial parameters and reported that the major influence on the strength of the aftereffect was the similarity between the spatial frequencies of the adaptation and test gratings. However, because of the nature of Harris's design and procedure, the three parameters were not independently varied during adaptation and testing. In the present experiment, the relative widths of the black bars and colored slits were varied independently during adaptation. In addition, the widths of the black bars and white slits also were varied independently during testing, which permitted direct assessment of the strength of the aftereffect. This design provided a context for separating more adequately the relative influence of bar width, slit width, and spatial frequency on the McCollough effect.

\section{METHOD}

\section{Subjects}

One hundred-ten college undergraduates served as Ss in the experiment. Participation in psychological research permitted Ss to earn extra credit in the psychology courses in which they were enrolled. Only Ss with normal red-green color vision were selected to serve in the experiment. Color anomalies were determined by using pseudoisochromatic plates (Hardy, Rand, \& Rittler, 1946).

\section{Stimuli and Procedure}

Adaptation consisted of repeated alternate exposure to two gratings. One grating had black bars and green slits (Wratten 61), and the other had black bars and magenta slits (Wratten 34A), counterbalanced between Ss so that either color grating could be vertical and the other horizontal. The adapting gratings were transilluminated in an Iconix Model 6131 three-channel tachistoscope. The photometric brightness was approximately $1.1 \mathrm{~mL}$ for the colored slits and $0.05 \mathrm{~mL}$ for the black bars. Each adapting grating subtended a visual angle of 4 deg vertically and $6 \mathrm{deg}$ horizontally. The total duration of the adaptation period was $12 \mathrm{~min}$, during which the alternating gratings were transilluminated for $2.5 \mathrm{sec}$ each, with dark interstimulus in tervals of $0.5 \mathrm{sec}$.

\section{Adaptation Conditions}

There were two basic experimental conditions in adaptation: 


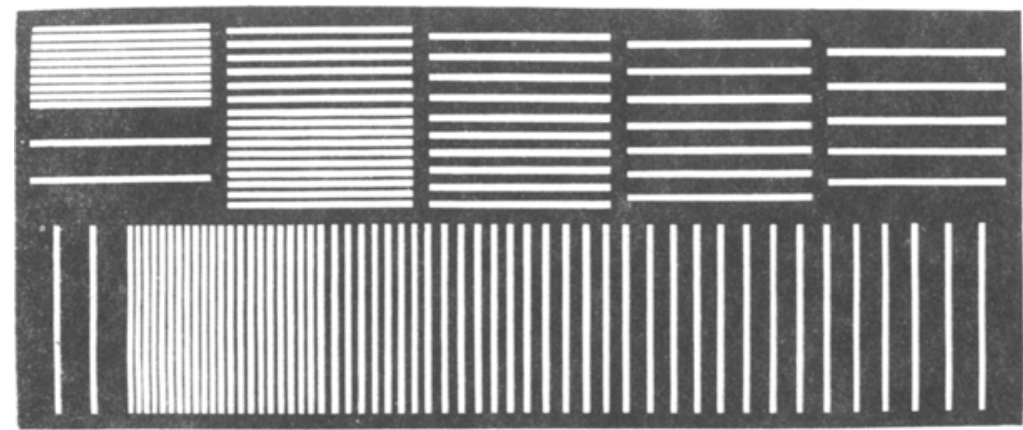

Fig. 1. Bar widths variable test (BVT) pattern.

(1) bar widths variable for adaptation (BVA), and (2) slit widths variable for adaptation (SVA). Within the BVA condition, there were five independent subgroups that received different pairs of adapting gratings. For all BVA groups, the widths of the colored slits subtended a standard visual angle of $11.25 \mathrm{~min}$. The widths of the black bars, however, varied, being either $0.5,1,2,3$, or 4 times the standard width (11.25 min). For the SVA groups, the relationship between the widths of the black bars and colored slits in the BVA condition was reversed. Here the black bars were always the standard width $(11.25 \mathrm{~min})$, and the widths of the colored slits were either $0.5,1,2,3$, or 4 times the standard width for the different subgroups. Because the group for which the widths of the bars and slits were equal $(1: 1)$ was the same for both the BVA and SVA conditions, there were nine independent experimental groups of $10 \mathrm{Ss}$. These groups differed in the relative widths of bars and slits used for the adapting gratings.

In addition to the BVA and SVA conditions, there was a control condition. The procedure for the control condition was identical to that of the experimental conditions except that each control $S$ was exposed to only one patternless colored field of either green or magenta. In addition, the display was dark during the intervals in which the alternate colored adapting grating was presented for the experimental conditions. The homogeneous colored field was green for one subgroup of $10 \mathrm{Ss}$ and magenta for another subgroup of 10 Ss. Thus, there were no striped grating patterns present in the control adaptation conditions. The purpose of this condition was to assess any potential differences regarding where colored afterimages appeared to be the strongest on the test patterns discussed below. For example, color aftereffects may appear to be differentially saturated in regions of the test gratings of different spatial extent or space-average luminance independent of the experimental manipulations. Such differences, both within and between test conditions, were assessed by having control Ss make judgments, similar to those required of $S s$ in the experimental conditions, regarding the strength of homogeneous color afterimages.

\section{Test Patterns}

Subsequent to exposure to the adapting stimuli, two separate test conditions (patterns), bar widths variable test (BVT) and slit widths variable test (SVT), were presented to all Ss. Each test pattern contained 10 different test gratings. These tests were designed to determine the relative effects of bar width, sit width, and spatial frequency on the color aftereffect. Figure 1 illustrates the BVT pattern. One white slit width was combined with each of 10 different black bar widths. In the BVT pattern the widths of all of the white slits subtended the same standard visual angle of $11.25 \mathrm{~min}$, while the widths of the black bars were $0.25,0.5$, and, thereafter, in 0.5 increments to 4.5 times the standard slit width.

Figure 2 illustrates the SVT pattern. The relationship between the widths of the black bars and white slits in the BVT pattern was reversed for the SVT pattern. In the SVT pattern the widths of all of the black bars subtended the same standard visual angle of $11.25 \mathrm{~min}$, while the widths of the white slits were $0.25,0.5$, and, thereafter, in 0.5 increments to 4.5 times the standard width. It can be seen in both Figs. 1 and 2 that the various test gratings were oriented both vertically and horizontally in each test pattern.

For each section (i.e., horizontal and vertical) of each test pattern (i.e., BVT and SVT) S was instructed to make an independent forced choice whether the slits of the gratings appeared to be more red or more green. The $S$ also was told to report in which part of each section these colors appeared to be the strongest or most saturated. The successive ratios of the widths of bars and slits for one orientation of the test gratings were spatially adjacent, while the ratios in the other orientation were not spatially adjacent. In order to eliminate this potential source of confounding, all Ss were tested twice with each section of each test pattern; for one series of judgments the section with the spatially adjacent ratios was oriented horizontally, and for the other it was oriented vertically. Thus, during testing each $S$ was required to make a series of eight forced-choice color naming judgments and eight judgments regarding where on the gratings the color af tereffect appeared to be the strongest.

\section{RESULTS}

The summary data for the nine experimental

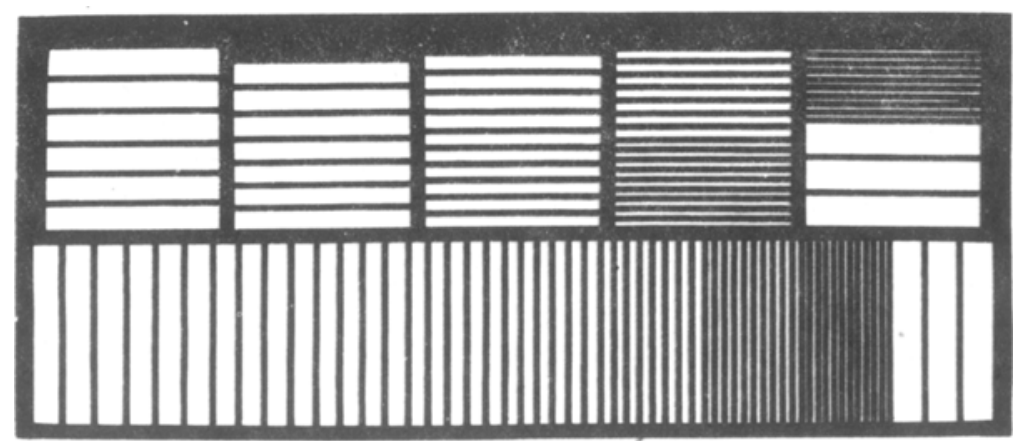

Fig. 2. Slit widths variable test (SVT) pattern. 
subgroups include (a) the percentage of "correct" color naming responses and (b) the mean widths of the relevant parameter (bars or slits) of the test gratings that produced the strongest color aftereffect for these correct judgments. A color naming response was scored correct for Ss in the experimental groups if it was the color associated with that orientation of a grating that would be consistent with the McCollough effect. The percent correct for the nine experimental groups ranged from $78 \%$ to $100 \%$, with a mean of $91 \%$. Percent correct did not appear to depend upon experimental conditions.

The criterion for correct color naming was not applicable to Ss in the control condition, who were presented a homogeneous field of either green or magenta during adaptation. Rather, the data for the two test patterns for the control condition merely reflect that grating ratio where the chosen color appeared strongest regardless of whether it was named green or red.

For purposes of clarity of exposition, the results regarding the gratings that produced the strongest color aftereffects will be presented separately for the bar widths variable adaptation (BVA) and the slit widths variable adaptation (SVA) conditions.

\section{BVA (Effects of the Black Bars)}

Figure 3 presents graphically the mean grating ratios that elicited the strongest aftereffects for the BVA subgroups and the control group (pooled over the two control subgroups) for both of the test conditions. This figure shows that all of the means for the BVA-SVT combinations were close to a ratio value of $1: 1$ and

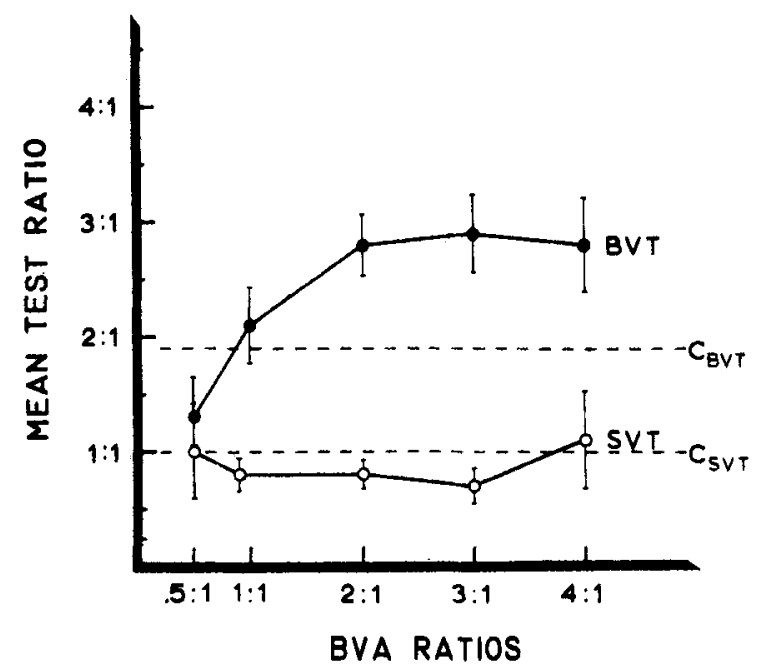

Fig. 3. Mean bar widths variable test (BVT) and slit widths variable test (SVT) gratings producing the strongest color aftereffects as a function of the bar widths variable adaptation (BVA) conditions, (95\% confidence levels are given for each mean.) The dashed lines labeled $\mathrm{C}_{\mathrm{BVT}}$ and $\mathrm{C}_{\mathrm{SVT}}$ indicate the mean BVT and SVT ratios producing the strongest color af terimages for $\mathrm{Ss}$ in the control conditions. (The 95\% confidence intervals for these two means are \pm .40 and \pm .53 , respectively.)

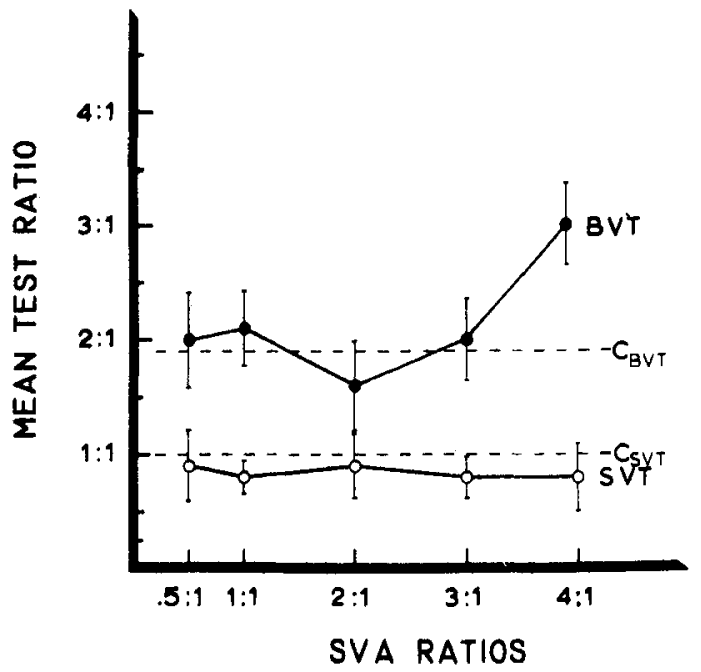

Fig. 4. Mean bar widths variable test (BVT) and slit width variable test (SVT) gratings producing the strongest color aftereffects as a function of the slit widths variable adaptation (SVA) conditions. (95\% confidence levels are given for each mean.) The dashed lines labeled $\mathrm{C}_{\mathrm{BVT}}$ and $\mathrm{C}_{\text {SVT }}$ indicate the mean BVT and SVT ratios producing the strongest oolor afterimage for $\mathrm{Ss}$ in the control conditions. (The 95\% confidence intervals for these two means are \pm .40 and \pm .53 , respectively.)

were not reliably different from each other. In addition, none of these individual means differed reliably from the mean SVT grating $(1: 1)$ designated by the control group. Trend analysis also indicated that there were no statistically significant components for the strength of the SVT aftereffects as a function of the widths of the black bars used during adaptation.

However, Fig. 3 shows that a different pattern of results obtained for the BVA subgroups with the BVT pattern. The mean BVT grating designated as producing the strongest aftereffect covaried with the BVA gratings. As the bar widths increased for the independent BVA exposure conditions, the bar widths that elicited the strongest aftereffects also increased. However, the mean bar width for maximal effect in the BVT pattern was not simply a linear function of the widths of the adapting bars. In addition to a statistically significant linear trend $[F(1,45)=23.47, p<.005]$, there was also a significant quadratic component for the BVA-BVT conditions $[\mathrm{F}(1,45)=6.61, \mathrm{p}<.025]$.

\section{SVA (Effects of the Colored Slits)}

Figure 4 shows that the relationship between the SVA subgroups and the designation of SVT gratings was similar to that obtained for the BVA-SVT combinations; viz, the mean SVT slit widths for maximal effect were close to a value of $1: 1$, independent of the widths of the adapting slits in the SVA subgroups. None of these individual means differed reliably from the mean SVT grating $(1: 1)$ designated by the control group. Furthermore, trend analysis indicated that there were no 
statistically significant components for the strength of the SVT aftereffects as a function of the widths of the colored slits used during adaptation.

Figure 4 also shows that the SVA subgroups of $.5: 1$, $1: 1,2: 1$, and $3: 1$ did not produce the same variation with the BVT pattern as did the BVA conditions with this test pattern. Rather, the means of these SVA subgroups did not differ reliably from each other nor from the mean value obtained for the control group with the same BVT measure. However, the mean BVT grating designated for the $4: 1$ SVA subgroup was $3: 1$, which was significantly higher than the means of the other SVA subgroups and significantly greater than the mean BVT grating $(1.9: 1)$ designated by the control group. There were significant linear $[F(1,45)=4.99, p<.05]$ and quadratic components $[F(1,45)=7.61, p<.01]$ for the SVA-BVT combinations.

\section{DISCUSSION}

The results suggest that the major determinant of pattern (orientation) contingent color aftereffects was the width of the black bars. Support for this conclusion was provided primarily by the finding that, as the width of the bars in the BVA gratings increased, the bar width of the BVT grating that produced the strongest aftereffect also increased. The strength of the aftereffect also appeared to approach an asymptote for BVA gratings with a ratio value of $2: 1$ and greater. A grating with a $2: 1$ ratio had a spatial frequency of 1.8 cycles/deg. Stromeyer (1972) reported a decline in the strength of the aftereffect at comparable low spatial frequencies. It may be the case that the same factors that produced the low frequency decline reported by Stromeyer placed a limit on the strength of the aftereffect at similar spatial frequencies in the present study.

It should be noted that, as the widths of the black bars increased for both BVA and BVT gratings, the spatial frequency of those gratings decreased. This same relationship also held between the widths of the slits and spatial frequency for the SVA and SVT gratings. In fact, spatial frequency was the same for any grating with the same ratio of widths of bars and slits, in both adaptation and testing, regardless of the relative widths of the bars and slits. Thus, the observed covariation between the BVA subgroups for the BVT pattern could have been due to variation of spatial frequency as well as that of the widths of the black bars. However, if spatial frequency were operating, adapting gratings of any given ratio (spatial frequency) should have produced similar effects for both the BVT and SVT patterns. Conversely, any difference in trends for width in the two test patterns must be attributable to something besides spatial frequency. It is clear in Fig. 3 that the two test conditions did not yield similar patterns of results for the BVA subgroups. Rather than covariation of any kind, the SVT grating ratios designated as producing the strongest aftereffect appeared unaffected by differences in the BVA conditions. These findings militate against the notion that the strength of the aftereffect was determined by spatial frequency.

The widths of the black bars was the same for all of the gratings in the SVT pattern. Thus, with the exception of the test gratings in which the widths of bars and slits were equal $(1: 1)$, it was not possible for Ss in the BVA subgroups to designate a SVT grating for which the widths of the black bars matched those of the adapting gratings. Therefore, if the major influence on the McCullough effect is, in fact, due to the width of the black bars, one would expect the finding of no systematic differences between the BVA subgroups for the SVT condition.

If the widths of the colored slits of the adapting gratings influence the strength of the aftereffect, one would expect results for the SVA-SVT conditions to be similar to those found for the BVA-BVT conditions. Examination of Fig. 4 reveals that such a relationship did not obtain. Instead, the slit widths in the test pattern producing maximal effect appear unaffected by adapting slit width. It is of interest to note that the mean slit width for all of the SVA conditions were close to the value $(1: 1)$ that matches the widths of the black bars for all of the SVA gratings.

The results of the SVA-BVT conditions do not lend themselves to unequivocal interpretation. As indicated in Fig. 4, the designated BVT gratings appear unaffected by the differences in the $5: 1,1: 1,2: 1$, and $3: 1$ SVA gratings. However, the mean grating ratio designated for the $4: 1$ SVA subgroup was significantly greater than the means of the other four subgroups in this condition. This difference was not readily interpretable in terms of variation of bar width, slit width, or spatial frequency.

The overall results of the present study were best described by the bar width hypothesis. It was therefore concluded that the width of the black bars was the major influence on the strength of pattern (orientation) contingent aftereffects. The conclusion of the present study was not in agreement with Harris's (1971) finding that spatial frequency was the major determinant of pattern (frequency) contingent aftereffects. However, there were substantial differences between the two studies that might account for these inconsistent findings. The two studies dealt with slightly different kinds of pattern contingent color aftereffects. Harris produced aftereffects by adapting Ss to two vertical gratings of different spatial frequencies. The high frequency grating had magenta slits, and the low frequency grating had green slits. The same pair of adapting gratings was used for all Ss, and, during testing, they were required to adjust the distance of a black and white vertical grating until the slits appeared to be colorless or "neutral." By comparing the results from three test gratings of different ratios of black bars to white slits, this neutral point was used to assess the relative influence of bar width, slit width, and spatial 
frequency. However, adjustment of the distance of each of these test gratings produced covariation of the bar width, slit width, and spatial frequency. Because of this covariation, it was not possible for Ss to match both bar width and slit width of the two asymmetrical test gratings (i.e., $3: 1$ and $1: 3$ ) halfway between the widths of the bars and slits of the symmetrical adapting gratings. Thus, effects specific to bar and slit widths would tend to cancel each other for the asymmetrical gratings. And, the neutral point for the symmetrical test grating $(1: 1)$ specific to halfway between the bar width and slit width of the adapting grating also would have to be halfway between the spatial frequencies of the adapting gratings.

Another difference between the present study and that of Harris's was the range of spatial frequencies of the adapting gratings. In the present study, the spatial frequency of the adapting gratings varied with the grating ratio and ranged from 1.1 (for a ratio of 5:1) to 3.6 (for a ratio of $4: 1$ ) cycles/deg of visual angle. On the other hand, Harris used one adapting condition with two gratings in the same orientation with spatial frequencies of 4.5 and $9.0 \mathrm{cycles} / \mathrm{deg}$. There is evidence suggesting that the parameters influencing grating specific aftereffects are different for gratings with spatial frequencies above 3 cycles/deg from those with spatial frequencies below 3 cycles/deg (Blakemore \& Campbell, 1969; Blakemore, Nachmias, \& Sutton, 1970).

Recordings from single neurons in the visual cortex of infrahumans have suggested that there are different types of orientation sensitive units (Hubel \& Wiesel, $1965,1968)$. For example, it has been found that some units are optimally sensitive to the specific widths of black bars in a particular orientation. Other units show similar specificity to the widths of light slits. The results of the present study suggest that the neurophysiological mechanism underlying the McCollough effect might consist of orientation specific units that are sensitive to both the widths of black bars and the chromatic characteristics of their surrounds as well. Both the "spatial-color" cells described by Dow and Gouras (1973) and the "opponent-color" cells described by Hubel and Wiesel (1968) would provide the requisites to serve these functions.

\section{REFERENCES}

Blackemore, C., \& Campbell, F. On the existence of neurones in the human visual system selectively sensitive to the orientation and size of retinal images. Journal of Physiology, $1969,203,237-260$.

Blakemore, C., Nachmias, J., \& Sutton, P. The perceived spatial frequency shift: Evidence for frequency selective neurones in the human brain, Journal of Physiology, 1970, 210, 727-750.

Dow, B. M., \& Gouras, P. Color and spatial specificty of single units in rhesus monkey foveal striate cortex. Journal of Neurophy siology, $1973,36,79-100$.

Hardy, L., Rand, G., \& Rittler, M. A screening test for defective red-green vision. Journal of the Optical Society of America. $1946,36,610.614$.

Harris, C. S. Effect of viewing distance on a color aftereffect specific to spatial frequency. Paper presented at the Psychonomic Society meeting, San Antonio, Texas, 1970.

Harris, C. S. Orientation-specific color aftereffects dependent on retinal spatial frequency rather than on stripe width. Journal of the Optical Society of America, 1971, 61, 689 (abstract).

Hubel, D. H., \& Wiesel, T. N. Receptive fields and functional architecture in two nonstriate areas (18 and 19) of the cat. Journal of Neurophysiology, 1965, 28, 229-280.

Hubel, D. H., \& Wiesel, T. N. Receptive fields and functional architecture of monkey striate cortex. Journal of Physiology, $1968,195,215-243$.

McCollough, C. Color adaptation of edge-detectors in the human visual sy stem. Science, 1965, 149, $1115-1116$.

Stromeyer, C. F. Further studies of the McCollough effect. Perception \& Psychophysics, 1969, 6, 105-110.

Teft, L. W., \& Clark, F. T. The effects of stimulus density on orientation specific aftereffects of color adaptation. Psychonomic Science, 1968, 11, 265-266.

(Received for publication July 20, 1973; revision received December 27,1973 .) 